\title{
Higgs signal gains strength
}

Latest analyses from the Large Hadron Collider boosts case for particle.

\section{Geoff Brumfiel}

07 February 2012

Today the two main experiments at the Large Hadron Collider (LHC), the world's most powerful particle accelerator, submitted the results of their latest analyses. The new papers boost the case for December's announcement of a possible Higgs signal, but let's not get too excited.

First, there are no new data in there - the LHC stopped colliding protons back in November, and these latest results are just rehashes of that earlier run. In the case of the Compact Muon Solenoid (CMS), physicists have been able to look at another possible kind of Higgs decay, and that allows them to boost their Higgs signal from 2.5 sigma to 3.1 sigma. Taken together with data from the other detector, ATLAS, Higgs' overall signal now unofficially stands at about 4.3 sigma. In other words, if statistics are to be believed, then this signal has about a $99.996 \%$ chance of being right.

It all sounds very convincing, but keep your hat on, because the fact is that statistical coincidences happen every day. Over at Cosmic Variance, Sean Carroll points out that there is a 3.8 sigma signal in the Super Bowl coin toss. Does that mean that

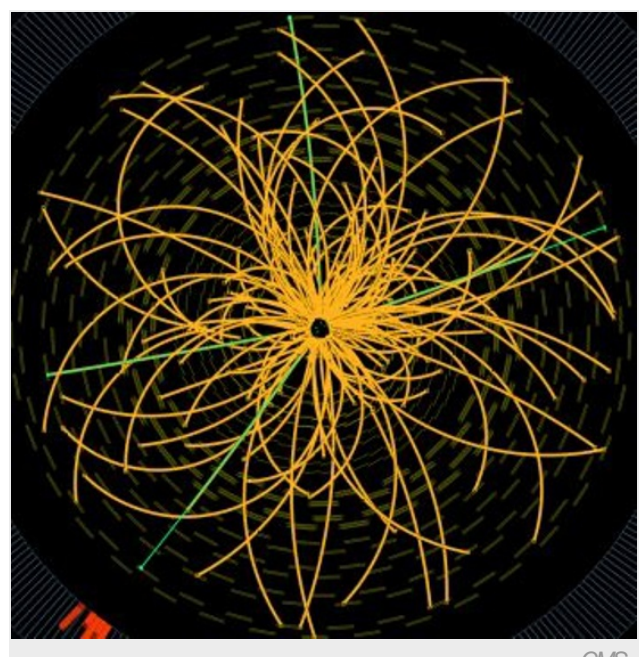

Reanalyses of existing data have pushed the overall Higgs signal up to $4.3 \sigma$.

they've discovered a super-partner to the bow? No. (If you don't get that joke, don't worry, it was written only as punishment for those who would.)

After the LHC starts again this spring, we'll be much closer to knowing what's actually going on. Right now, scientists are meeting in Chamonix, France, to decide at what power to run the collider this coming year. The latest rumours are that the machine will push from 7 to 8 teraelectronvolts, and it will also increase its luminosity (the number of collisions per pass).

For a little more context about what's going on, check out this video of my trip back in November:

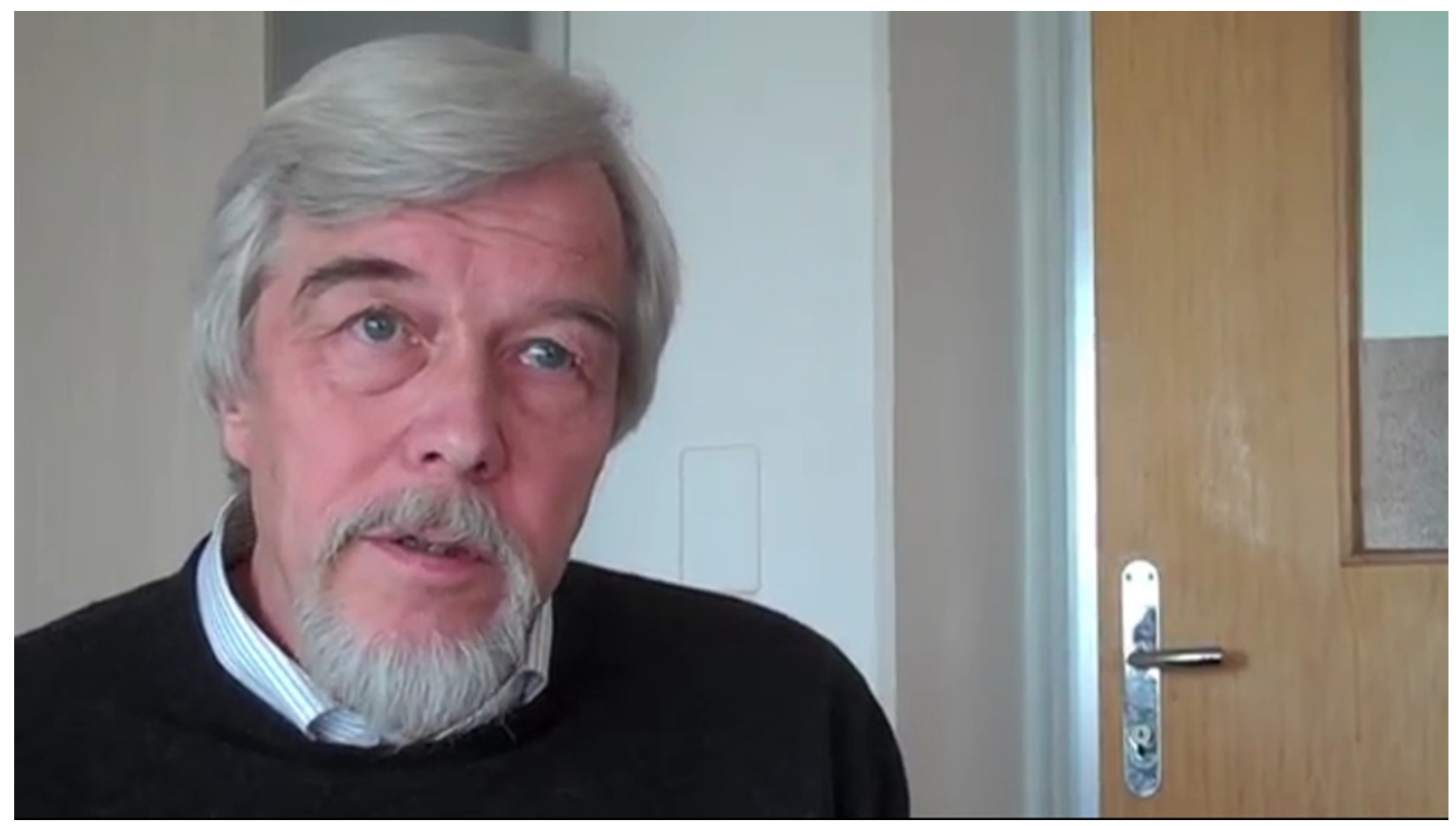


Nature | doi:10.1038/nature.2012.9992 